\title{
Human herpesvirus 6 and multiple sclerosis: survey of anti-HHV-6 antibodies by immunofluorescence analysis and of viral sequences by polymerase chain reaction
}

\author{
Patrizia Sola, Elisa Merelli, Roberto Marasca, Maria Poggi, Mario Luppi, \\ Marcello Montorsi, Giuseppe Torelli
}

\begin{abstract}
A possible involvement of human herpesvirus 6 (HHV-6) infection in the pathogenesis of multiple sclerosis (MS) was investigated. The immunofluorescence analysis of sera from $126 \mathrm{MS}$ patients showed significantly higher antiHHV-6 antibody titres in MS sera than in 500 normal controls. A polymerase chain reaction (PCR) assay of the peripheral blood mononuclear cell (PBMC) DNAs of $31 \mathrm{MS}$ patients and 24 normal subjects was positive in one normal control and in one MS patient. The Southern blot analysis indicated an unexpectedly high level of viral sequences in the MS patient, but not in the control. Since viral sequences are rarely present in MS subjects, the high anti-HHV-6 antibody titres found in MS are likely to be related to immune impairment rather than reactivation of a latent infection.
\end{abstract}

(F Neurol Neurosurg Psychiatry 1993;56:917-919)

Human herpesvirus 6 (HHV-6) was first isolated in 1986 from six patients with lymphoproliferative disorders, two of whom also had HIV antibodies. ${ }^{1}$ This virus is selectively cytopathic to freshly cultured CD4 + T lymphocytes, although it may be infectious at lower levels for other cell lines, including B cells, macrophage/monocytes, and glial cells. ${ }^{23}$ HHV- 6 is the causative agent of the exanthem subitum of children, 4 while the adult primary infection has been related to chronic fatigue syndrome. ${ }^{5}$ The infection is widespread, its seroprevalence in healthy adult populations ranging between $50 \%$ and $85 \% .^{6-11}$ Seroconversion occurs early in childhood, and the antibody titres decrease in a normal population after age 40.12 HHV-6 sequences have been detected by polymerase chain reaction (PCR) in saliva samples and in mononuclear cells of the peripheral blood of healthy adults, ${ }^{13}$ suggesting that the viral genome may persist in the oropharynx and that normally only rare cells in the peripheral blood of healthy individuals are infected. HHV-6 is frequently found, and sometimes isolated, in HIV infected patients. ${ }^{14}$ Since this herpesvirus induces CD4 receptor, ${ }^{14}$ is able to transactivate the HIV long terminal repeat, ${ }^{15}$ and to productively coinfect human $T$ cells with $\mathrm{HIV},{ }^{16}$ a possible role in the pathogenesis of AIDS has been suggested.

High titres of anti-HHV-6 antibodies and the presence of HHV-6 genomic sequences are frequently associated with immune disorders. ${ }^{17} \mathrm{Up}$ to now, a large number of viral agents have been investigated in multiple sclerosis (MS), ${ }^{18}$ but not HHV-6. We were interested in studying the HHV-6 infection in MS patients because of its biological characteristics, including its strong CD4 $+\mathrm{T}$ cell tropism, synergic action with other viruses, and the occurrence of the infection early in human life with persistence in latent form in the adults.

\section{Materials and methods}

We tested for anti-HHV-6 antibody by indirect immunofluorescence analysis (IFA) the serum and CSF samples collected from 126 MS patients (47 men and 79 women, mean age 35 years, range 13-70). The sera from 500 healthy, HIV-negative, blood donors (250 men and 250 women, mean age 44 years) were also examined to establish a control value for a normal population. Indirect IFA was performed on HHV-6 infected HSB2 cells as described by Ablashi et al. ${ }^{19}$ Serum samples were examined at 1:40, 1:80 and 1:160 dilutions. CSF samples were examined undiluted and diluted 1:40 and 1:80. Antibody titration was performed double blind by two operators.

PCR analysis was performed according to Torelli et $a l^{10}$ in the DNAs extracted from the peripheral blood mononuclear cells (PBMCs) of $31 \mathrm{MS}$ patients and of 24 blood donors. The oligonucleotides used as primers and probes were synthesised on an automated solid phase synthesiser (Applied Biosystems Inc, Mod 381A), by standard phosphoramidite chemistry. Two sets of primers and probes were used. One set, representing a portion of the gene for the major capsid protein, ${ }^{20}$ has the following sequence: (1) 5'ATTAGGACCGATCGGCTCTA3' as sense primer, (2) 5'GTGTAGGTGGTCGAATGCGA3' as antisense primer, (3) 
5' TCCCGGCGTTCACGGTGAATTGAGTA3' as internal probe. The second set, representing a portion of the $\mathrm{ZVH} 14$ segment, ${ }^{21}$ has the following sequence: (1) 5'CCCATTTACGATTTCCTGCACCACCTCTCTGC $3^{\prime}$ as sense primer, (2) 5'TTCAGGGACCGTTATGTCATTGAGCATGTCG3' as antisense primer, (3) 5 ' C CGTAAAAAAT T TACACCTCCATTTCATCTT3' as internal probe. Thirty cycles of amplification were performed with an automatic thermal cycler (MJ Research, Cambridge, MA, USA). The amplification products, a 300 nucleotide segment for the first set and a 186 nucleotide segment for the second set, were then subjected to electrophoresis, transferred to a nylon membrane by vacuum blotting, and hybridised with an oligonucleotide probe end-labelled with $\gamma{ }^{32} \mathrm{P}-\mathrm{ATP}$. The autoradiography was then performed for 2 and 7 days at $-80^{\circ} \mathrm{C}$. A positive control was represented by HHV-6 infected HSB-2 DNA. Two negative controls (a human placental DNA and a reaction mixture without DNA) were used for each group of 10 samples examined. In Southern blot analysis the pZVH14 plasmid described by Josephs et al ${ }^{21}$ was used as probe.

\section{Results}

We found significantly higher anti-HHV-6 IgG antibody titres in MS patients in comparison with the blood donors group at IFA analysis (table). Data obtained with sera at 1:20 dilution were disregarded because of the non-specific immunofluorescence detected in control uninfected cells.

Only one out of 31 MS DNA samples showed a strong signal with both sets of primers in the PCR analysis. The same sample was positive when analysed by Southern blot technique using as probe the pZVH14 plasmid, containing $9 \mathrm{~kb}$ of the HHV-6 genome including the segment amplified by one set of primers. To avoid false positive results due to possible contaminations, both PCR and Southern analysis were performed on three different DNA samples extracted from cell pellets collected at different intervals during a remission phase of the disease. The DNA of the positive patient was digested with Eco RI, Hind III and Bam HI restriction enzymes and hybridised with the pZVH14 plasmid. Then it was compared with the DNAs extracted from normal human pla-

Table Presence of HHV-6 antibody at IFA analysis in the two groups of subjects: multiple sclerosis (MS) and blood donors (BD)

\begin{tabular}{llll}
\hline & $\begin{array}{l}M S \\
(n=126)\end{array}$ & $\begin{array}{l}B D \\
(n=500)\end{array}$ & $\chi^{2}$ \\
\hline Sample dilution: & & & \\
serum $1: 40$ & $74 \%$ & $41 \%$ & $42 \cdot 1^{\star}$ \\
serum $1: 80$ & $49 \%$ & $18 \%$ & $51 \cdot 6^{\star}$ \\
serum $1: 160$ & $23 \%$ & $4 \%$ & $44 \cdot 7^{\star}$ \\
CSF undiluted & $7 \%$ & nd & \\
CSF 1:40 & $2 \%$ & nd & \\
CSF 1:80 & $1 \%$ & nd & \\
\hline
\end{tabular}

nd $=$ not determined. ${ }^{\star} \mathrm{p}<0.0005$. centa and from HSB-2 cells infected by HHV-6, strain GS. The pZVH14 sequence of this case compared with the same sequence of GS strain, showed the same restriction fragment length polymorphism (RLFP), with all the enzymes used. The PCR assay performed on the DNAs of 24 normal subjects showed only one positive case. In this case the signal was very faint and the Southern analysis was negative, suggesting that the number of copies of HHV-6 sequences detected is much lower than in the positive MS patient.

Both the two PCR positive cases were negative for serum anti-HHV-6 antibody at IFA analysis.

\section{Discussion}

Our results on anti-HHV-6 antibody titres in the blood donors group are in good agreement with published data, ${ }^{7-9}$ and suggest that HHV-6 infection is widespread in our region, as well as in a number of other countries. Moreover, the use of 1:40 dilution makes unlikely a non-specific reaction, while a possible immunological crossreactivity between HHV-6 and other herpesviruses has been ruled out by several authors. ${ }^{189}$

To explain the high anti-HHV-6 antibody titres in MS, it may be speculated that in this disease the impairment of the cellular immune response may lead to the reactivation of an HHV-6 latent infection. This reactivation may in turn determine an increase of anti-HHV-6 antibody titres. This explanation cannot be ruled out; however, it seems unlikely in view of our PCR data, since we found HHV-6 sequences in the PBMC DNA of only one out of $31 \mathrm{MS}$ patients, in spite of the known lymphotropism of this virus. In the absence of a satisfactory explanation we can only say that the immune system in MS is activated against $\mathrm{HHV}-6$, in the absence of detectable viral sequences.

In nine MS patients anti-HHV-6 IgG were present also in the CSF. Because only two out of nine MS patients with CSF anti-HHV6 antibodies show BBB damage, we suggest that in these patients the immune stimulation leads to the synthesis of these antibodies by intrathecal lymphocyte clones, as confirmed by the high CSF IgG index frequently found. Within the limits of sensitivity of our PCR assay, two positive cases were found: one MS patient and one healthy control, both negative for HHV-6 antibody. We have no explanation for this discrepancy which is typically associated with latent infection ${ }^{22}$ or could be due to immune disregulation. In spite of the use of two different sets of primers, the molecular tests performed do not allow us to establish with absolute certainty the presence in PBMCs of complete HHV-6 genomes. However, the amplified sequences are HHV6 specific, because the primers used do not cross hybridise with normal human DNA or with other herpesvirus DNAs.

The positive MS patient showed an unexpectedly high number of copies of HHV-6 sequences, so that these sequences were easily 
detectable by a standard Southern analysis, contrary to the normal positive control. Only an in situ hybridisation assay would allow us to establish if there are few cells with a high number of HHV- 6 sequences or several cells carrying few HHV-6 molecules. The faint positivity in the PCR assay of the normal positive subject suggests that in this case only a rare cell in the peripheral blood is infected with few copies of HHV-6. The difference between the two positive cases could be related to the immune impairment of MS, which would make possible the expansion of a latent infection not allowed by an intact immune system. Otherwise, the immune impairment of the disease could be viewed as a consequence rather than as the origin of the high viral titres.

Since HHV-6 is able to infect other cells beside $T$ lymphocytes, a reason for these results may be that the virus remains latent in cells other than PBMCs, perhaps in the oligodendrocytes. Lastly, it is also impossible to exclude that variant HHV-6, not well hybridising to our primers, can be present in the PBMCs examined.

At present, we cannot draw any conclusion in favour of or against a possible role of HHV-6 in the pathogenesis of some MS cases. Moreover, the possibility that HHV-6 proteins may cause the transactivation of other cellular or viral genes contributing in this way to the immune derangement of MS cannot be ruled out. ${ }^{23} \mathrm{We}$ emphasise that further refining of antibody test systems, experience with larger numbers of subjects, and careful prospective and molecular studies are necessary before conclusions can be made regarding the relations between the MS and HHV-6 infection.

This work was supported by a grant and a fellowship (PS) from the Associazione Italiana Sclerosi Multipla (AISM) and by ICSC-World Laboratory, Geneva, as part of project MCD2.

1 Salahuddin SZ, Ablashi DV, Markham PD, et al. Isolation of a new virus, HBLV, in patients with lymphoproliferative disorders. Science 1986;234:596-600.

2 Lusso $\mathrm{P}$, Markham PD, Tschachler E, et al. In vitro cellular tropism of human B-lymphotropic virus (huma herpesvirus 6). $f$ Exp Med 1988;167:1659-70.

3 Takahashi K, Sonoda S, Higashi K, et al. Predominant CD4 T-lymphocyte tropism of human herpesvirus 6related virus. F Virol 1989;63:3161-3.

4 Yamanishi K, Okuno T, Shiraki K, et al. Identification of human herpesvirus- 6 as a causal agent for exanthem subitum. Lancet 1988;i:1065-7.

5 Krueger GRF, Koch B, Ablashi DV. Persistent fatigue and depression in patient with antibody to human Blymphotropic virus. Lancet 1988;ii:817.

6 Ablashi DV, Josephs SF, Buchbinder A, et al. Human Blymphotropic virus (human herpesvirus 6). $\mathcal{F}$ Virol Meth 1988;21:29-48.

7 Linde A, Dahl H, Wahren B, Fridell E, Salahuddin SZ, Biberfeld P. IgG antibodies to human herpesvirus 6 in Biberfeld $P$. IgG antibodies to human herpesvirus 6 in
children and adults in primary Epstein-Barr virus and cytomegalovirus infections. $f$ Virol Meth 1988;21: cytomegalo

8 Saxinger C, Polesky H, Eby N, et al. Antibody reactivity with HBLV (HHV-6) in US population. $f$ Virol Meth 1988;21:199-208.

9 Okuno T, Takahashi K, Balachandran K, et al. Seroepidemiology of human herpesvirus 6 infection in normal children and adults. $\mathcal{f}$ Clin Microbiol 1989;27: 651-3.

10 Torelli G, Marasca R, Luppi M, et al. Human herpes virus $6(\mathrm{HHV}-6)$ in human lymphomas: identification of specific sequences in Hodgkin's lymphomas by polymerase chain reaction (PCR) Blood 1991;77:2251-8.

11 Merelli E, Sola P, Faglioni P, Poggi M, Montorsi M, Torelli G. Newest human herpesvirus (HHV-6) in the Torelli G. Newest human herpesvirus (HHV-6) in the eases. Acta Neurol Scand 1992;85:334-6.

12 Brown NA, Sumaya CV, Liu CR, et al. Fall in human herpesvirus 6 seropositivity with age. Lancet 1988;i:396.

13 Jarrett RF, Clark DA, Josephs SF, Onions DE. Detection of human herpesvirus 6 DNA in peripheral blood and saliva. ₹ Med Virol 1990;32:73-6.

14 Lusso $\mathrm{P}$, De Maria A, Malnati M, et al. Induction of CD4 and susceptibility to HIV infection in human CD8 $+\mathrm{T}$ lymphocytes by human herpesvirus 6. Nature 1991; 349:533-5.

15 Horvat RT, Wood C, Balachandran N. Transactivation of human immunodeficiency virus promoter by human herpesvirus 6. F Virol 1989;63:970-3.

16 Lusso, P, Ensoli B, Markham PD, et al. Productive dual infection of human CD4 + T lymphocytes by HIV-1 infection of human CD4 + T lymph
and HHV-6. Nature 1989;337:370-3.

17 Biberfeld P, Petren AL, Eklund A, et al. Human herpesvirus 6 (HHV-6, HBLV) in sarcoidosis and lymphoproliferative disorders. $\mathcal{F}$ Virol Meth 1988;21:49-59.

18 Johnson RT. Viral aspects of multiple sclerosis. In: Koetsier JC, ed. Handbook of clinical neurology. Amsterdam: Elsevier Science, 1985;47:319-36.

19 Ablashi DV, Lusso P, Hung CL, et al. Utilization of human hematopoietic cell lines for the propagation and characterization of HBLV (human herpesvirus 6). Int $\mathcal{F}$ Cancer 1988;42:787-91.

20 Littler E, Lawrence G, Kiu MY, Barrell BG, Arrand JR. Identification, cloning and expression of the major capsid protein gene of human herpesvirus $6 . \mathcal{F}$ Virol 1990;64:714.

21 Josephs SF, Salahuddin SZ, Ablashi DV, Schachter F, Wong-Staal F, Gallo RC. Genomic analysis of the human B-lymphotropic virus (HBLV). Science 1986; 234:601-3.

22 Krueger GRF, Manak M, Burgeois N, et al. Persistent active herpesvirus infection associated with atypical policlonal lymphoproliferation (APL) and malignant lymphoma. Anticancer Res 1989;9:1457-76.

23 Thompson BJ, Efstathiou S, Honess RW. Acquisition of the human adeno-associated virus type- 2 reg gene by human herpesvirus type 6. Nature 1991;351:78-80. 